\title{
BOUNDS ON MEAN EXIT TIME FOR PURELY TIME-DEPENDENT DRIFT AND DIFFUSION
}

\author{
EDWARD ALLEN \\ Department of Mathematics and Statistics \\ Texas Tech University \\ Lubbock, TX 79409 USA
}

\begin{abstract}
The mean first exit time is studied for the one-dimensional problem with one-sided exit and purely time-dependent drift and diffusion. Exact mean exit times are derived when the drift coefficient is constant and the diffusion coefficient is bounded. When the drift and diffusion coefficients both vary with time, bounds on mean first exit time are derived. Examples are described that illustrate the usefulness of the results.
\end{abstract}

AMS Subject Classification: 60H10, 92D25, 34F05

Received: June 13, 2017; Accepted: October 31, 2018;

Published: November 13, 2018. doi: 10.12732/caa.v22i4.7

Dynamic Publishers, Inc., Acad. Publishers, Ltd. http://www.acadsol.eu/caa

\section{MEAN FIRST EXIT TIME PROBLEM}

Mean first exit time, or mean first passage time, is useful in many areas including mathematical biology, structural dynamics, nuclear engineering, and mathematical finance. In structural dynamics, failure of a mechanical system due to random vibrations is a first passage problem [16]. The optimum time to sell an asset is an example of a first exit time problem in finance [8]. In biology, the persistence time, or extinction time, is a first exit time problem $[1,5,6]$. Another application of exit time in biology is in development of hu- 
man life table data [19]. In addition, the power doubling time for a subcritical system is a first-passage time problem in nuclear engineering [3].

To derive exact mean exit times is difficult even for the simplest problems. In the present investigation, the mean exit time is studied for the onedimensional problem with one-sided exit and purely time-dependent drift and diffusion. Exact probability densities can be obtained for special cases of this problem [15], however, in general, the mean exit time must be determined computationally. Bounds on mean exit time allow greater understanding of many problems and bounds may be sufficient in studies of certain problems. In the present investigation, exact mean exit times are derived when the drift coefficient is constant and the diffusion coefficient is bounded. When the drift and diffusion coefficients both vary with time, bounds on mean exit time are derived.

The exit time problem examined in the present investigation is defined by the Itô stochastic differential equation

$$
d x(t)=a(t) d t+b(t) d W(t)
$$

where $W(t)$ is a standard Wiener process, $x(0)=0$, and exit occurs at the first time $t_{e}$ when $x\left(t_{e}\right)=x_{e}$ where $x_{e}$ is a given fixed positive number. Of interest, in the present investigation, is the mean first exit time $T_{e}=\mathbb{E}\left(t_{e}\right)$. It is assumed that the drift coefficient $a(t)>0$ for $t>0$ and that the drift and diffusion coefficients, $a(t)$ and $b(t)$, are bounded and have bounded continuous first derivatives for $t \in(0, \infty)$.

The mean exit time problem can be studied by considering the reliability function (or survival probability), $R(y, t)$, which satisfies the backward Kolmogorov equation $[2,10,16,18]$ associated with (1.1). This equation has the form

$$
\frac{\partial R(y, t)}{\partial t}=a(t) \frac{\partial R(y, t)}{\partial y}+\frac{b^{2}(t)}{2} \frac{\partial^{2} R(y, t)}{\partial^{2} y}
$$

with $R\left(x_{e}, t\right)=0$ for $t>0$ and $R(y, 0)=1$ for $y<x_{e}$. The reliability function, $R(y, t)$, is the probability that the exit time is greater than $t$ assuming initially that $x(0)=y$. (That is, $R(y, t)$ is the probabilty that trajectories starting at position $y$ do not exit before time $t$.) If the reliability function is known, the mean exit time for problem (1.1) can be obtained by integrating the reliability function over time $[2,18]$. In particular, when the initial position is $x(0)=0$ 
such as for problem (1.1), then

$$
T_{e}=\int_{0}^{\infty} R(0, t) d t
$$

It is useful to define the parameter $\gamma(t)$ as

$$
\gamma(t)=\frac{a(t)}{\frac{1}{2} b^{2}(t)} .
$$

Furthermore, the function $h$ is defined so that if $\int_{0}^{t} a(z) d z=\tau$ then $h(\tau)=t$. If $\gamma(t)$ is constant, then the exact probability density of trajectories to (1.1) is given explicitly by Molini, et al. [15]. The following proposition shows that, when $\gamma(t)$ is constant, the mean exit time is equal to a certain integral involving an inverse Gaussian probability density.

Proposition 1.1. If $\gamma(t)=q$ where $q$ is a constant, then the mean exit time for (1.1) satisfies

$$
T_{e}=\int_{0}^{\infty} h(\tau) p\left(\tau ; x_{e}, q\right) d \tau
$$

where $p\left(\tau ; x_{e}, q\right)$ is the inverse Gaussian or Wald density [9] with mean $x_{e}$ and variance $2 x_{e} / q$ given explicitly by

$$
p\left(\tau ; x_{e}, q\right)=\left(\frac{q x_{e}^{2}}{4 \pi \tau^{3}}\right)^{1 / 2} \exp \left(\frac{-q\left(\tau-x_{e}\right)^{2}}{4 \tau}\right) .
$$

Proof. For $\gamma(t)=q$, a variable change in (1.2) simplifies the backward Kolmogorov equation for the reliability function. Letting $\tau=\int_{0}^{t} a(z) d z$, then (1.2) can be written as

$$
\frac{\partial R(y, \tau)}{\partial \tau}=\frac{\partial R(y, \tau)}{\partial y}+\frac{1}{q} \frac{\partial^{2} R(y, \tau)}{\partial^{2} y} .
$$

Backward Kolmogorov equation (1.7) corresponds to the exit time problem in variable $\tau$ :

$$
d z(\tau)=d \tau+\sqrt{2 / q} d W(\tau)
$$

with $z(0)=0$ and exit occuring when $z\left(\tau_{e}\right)=x_{e}$. Exit time problem (1.8), with constant drift and diffusion coefficients, has well-known properties [9]. The mean exit time for (1.8) is $x_{e}$ and the probability density of exit times is 
given by the Wald density with mean $x_{e}$ and variance $2 x_{e} / q$. As $t=h(\tau)$, it follows that

$$
x_{e}=\mathbb{E}\left(\int_{0}^{t_{e}} a(z) d z\right) \text { and } T_{e}=\int_{0}^{\infty} h(\tau) p\left(\tau ; x_{e}, q\right) d \tau .
$$

For example, if $a(t)=\mu>0$ and $b(t)=\sigma$ are constants, then the mean exit time for problem (1.1) is exactly equal to $T_{e}=x_{e} / \mu$. Notice, in this case, when $a(t)$ and $b(t)$ are constants, the reliability function for (1.1) is the wellknown Wald distribution or inverse-Gaussian distribution [9]. In addition, when $a(t)=\mu$ and $b(t)=\sigma$, the solution, $p(x, t)$, of the forward Kolmogorov equation for the probability density function of the trajectories of (1.1) is given by $[15]$ :

$$
\begin{aligned}
& p(x, t)= \\
& \quad \frac{\exp \left(\frac{-(x-\mu t)^{2}}{2 \sigma^{2} t}\right)-\exp \left(\frac{2 x_{e} \mu}{\sigma^{2}}\right) \exp \left(\frac{\left.-\left(-x+2 x_{e}+\mu t\right)^{2}\right)}{2 \sigma^{2} t}\right)}{\sqrt{2 \pi \sigma^{2} t}} .
\end{aligned}
$$

Other than when $a(t)=\mu$ and $b(t)=\sigma$, it appears that the exact mean exit time is generally not known for problem (1.1) and numerical procedures must be employed to computationally solve (1.1), (1.2), or numerically integrate the reliability function if it is known.

In the next section, the result in Proposition 1.1 is extended to include the case when the drift coefficient $a(t)$ is constant but the diffusion coefficient $b(t)$ varies with $t$. Then, bounds are derived for the general exit-time problem (1.1) when both coefficients depend on time $t$. Finally, before summarizing the investigation, several examples are described that illustrate the results.

\section{BOUNDS ON MEAN FIRST EXIT TIME}

Bounds for the mean exit time of solutions to (1.1) are given in this section. First, the following simple result is useful.

Proposition 2.1. Consider the following two SDE exit problems of the form (1.1) with the same realization of $W(t)$ :

$$
d \hat{x}(t)=\hat{a}(t) d t+b(t) d W(t) \quad \text { and } \quad d \tilde{x}(t)=\tilde{a}(t) d t+b(t) d W(t),
$$


with $\hat{x}(0)=\tilde{x}(0)=0$,

and exit occurring when the trajectories reach $x_{e}>0$. Let $\hat{T}_{e}$ and $\tilde{T}_{e}$ be the corresponding mean exit times. If $0<\tilde{a}(t) \leq \hat{a}(t)$ for $t \geq 0$, then $\hat{T}_{e} \leq \tilde{T}_{e}$.

Proof. Subtracting the two SDEs yields

$$
d(\hat{x}(t)-\tilde{x}(t))=(\hat{a}(t)-\tilde{a}(t)) d t \quad \text { with } \quad \hat{x}(0)-\tilde{x}(0)=0 .
$$

As $(\hat{a}(t)-\tilde{a}(t)) \geq 0$ for $t \geq 0$, the solution of (2.1) implies that $\hat{x}(t) \geq \tilde{x}(t)$ for $t \geq 0$. Each trajectory of the first SDE therefore exits before or at the same time as the corresponding trajectory of the second SDE. Thus, $\hat{T}_{e} \leq \tilde{T}_{e}$.

The next theorem gives the exact mean exit time when the drift coefficient $a(t)=\mu$ is constant and the diffusion coefficient $b(t)$ is bounded on $(0, \infty)$.

Theorem 2.2. Suppose that $a(t)=\mu$ and $b(t)$ is bounded and has a bounded continuous derivative for $t \in(0, \infty)$. Then, the mean exit time, $T_{e}$, is equal to $x_{e} / \mu$.

Proof. First, the result clearly holds if $b(t)=\sigma$ for $t>0$. Now, in a second case, suppose that $b(t)$ is piecewise constant, specifically,

$$
b(t)= \begin{cases}\sigma_{1}, & 0<t<t_{1} \\ \sigma_{2}, & t>t_{1}\end{cases}
$$

where $\sigma_{1}$ and $\sigma_{2}$ are constants. Let $p\left(x, t_{1}\right)$ be the probability density of the particles at position $x$ and time $t_{1}$ and let

$$
P_{i}\left(t_{1}\right)=\int_{x_{i}}^{x_{i+1}} p\left(x, t_{1}\right) d x \approx p\left(x_{i}, t_{1}\right) \Delta x
$$

be the proportion of particles in $\left[x_{i}, x_{i+1}\right]$ at time $t_{1}$ where $x_{i+1}-x_{i}=\Delta x$ is small. Let $T_{i}\left(t_{1}\right)$ be the average time to exit given the particle starts at time $t_{1}$ from position $x_{i}$. As $\sigma_{2}$ is constant and $t_{1}$ has already elapsed, the average exit time satisfies $T_{i}\left(t_{1}\right)=t_{1}+\left(x_{e}-x_{i}\right) / \mu$ which follows directly from the first case. Finally, let $\bar{T}_{e}\left(0, t_{1}\right)$ be the mean exit time of the particles that have exited up to time $t_{1}$ and $\bar{P}_{e}\left(0, t_{1}\right)$ be the proportion of particles that have exited during this time interval. Therefore, the mean exit time, $T_{e}$, approximately satisfies

$$
T_{e} \approx \bar{T}_{e}\left(0, t_{1}\right) \bar{P}_{e}\left(0, t_{1}\right)+\sum_{i=1}^{\infty} P_{i}\left(t_{1}\right) T_{i}\left(t_{1}\right)
$$


and as $\Delta x \rightarrow 0$,

$$
T_{e}=\bar{T}_{e}\left(0, t_{1}\right) \bar{P}_{e}\left(0, t_{1}\right)+\int_{-\infty}^{x_{e}} p\left(x, t_{1}\right)\left(t_{1}+\left(x_{e}-x\right) / \mu\right) d x .
$$

However, if $\sigma_{2}=\sigma_{1}$, then by the first case the identical equation holds but, in this case, $T_{e}$ is exactly $T_{e}=x_{e} / \mu$. This implies that $T_{e}=x_{e} / \mu$ also when $b(t)=\sigma_{1}$ for $t<t_{1}$ and $b(t)=\sigma_{2}$ for $t>t_{1}$.

Now consider a third case where

$$
b(t)=\left\{\begin{array}{cc}
\sigma_{1}, & 0<t<t_{1} \\
\sigma_{2}, & t_{1}<t<t_{2} \\
\sigma_{3} & t_{2}<t<t_{3}
\end{array}\right.
$$

Let $p\left(x, t_{2}\right)$ be the probability density of the particles at position $x$ and time $t_{2}$ for this third case and let

$$
P_{i}\left(t_{2}\right)=\int_{x_{i}}^{x_{i+1}} p\left(x, t_{2}\right) d x \approx p\left(x_{i}, t_{2}\right) \Delta x
$$

be the proportion of particles in $\left[x_{i}, x_{i+1}\right]$ at time $t_{2}$ where $x_{i+1}-x_{i}=\Delta x$ is small. Let $T_{i}\left(t_{2}\right)$ be the average time to exit given the particle starts at time $t_{2}$ from position $x_{i}$. As $\sigma_{3}$ is constant and $t_{2}$ has already elapsed, the mean time is $T_{i}\left(t_{2}\right)=t_{2}+\left(x_{e}-x_{i}\right) / \mu$. Finally, let $\bar{T}_{e}\left(0, t_{2}\right)$ be the mean exit time of the particles that have exited up to time $t_{2}$ and $\bar{P}_{e}\left(0, t_{2}\right)$ be the proportion of particles that have exited during this time interval. Then, the mean exit time for the exit problem approximately satisfies

$$
T_{e} \approx \bar{T}_{e}\left(0, t_{2}\right) \bar{P}_{e}\left(0, t_{2}\right)+\sum_{i=1}^{\infty} P_{i}\left(t_{2}\right) T_{i}\left(t_{2}\right)
$$

and as $\Delta x \rightarrow 0$,

$$
T_{e}=\bar{T}_{e}\left(0, t_{2}\right) P_{e}\left(0, t_{2}\right)+\int_{-\infty}^{x_{e}} p\left(x, t_{2}\right)\left(t_{2}+\left(x_{e}-x\right) / \mu\right) d x .
$$

However, it is known by referring to the second case, that if $\sigma_{3}=\sigma_{2}$, then the same equation holds but $T_{e}$ is exactly $T_{e}=x_{e} / \mu$. Thus, $T_{e}=x_{e} / \mu$ as well for the third case.

This argument can be repeated again and again to show that if the diffusion coefficient $b(t)$ is piecewise constant and bounded and provided that the drift 
coefficient $a(t)$ is constant, then $T_{e}=x_{e} / \mu$. Now, for example, if the EulerMaruyama numerical method $[10,12,13]$ is applied to problem (1.1), where $a(t)$ and $b(t)$ are bounded and have bounded continuous first derivatives, then it is known that the numerical method converges to the exact mean exit time $[7,11]$. However, the Euler-Maruyama method is the same whether applied to the continuous function $b(t)$ or to a piecewise constant approximation of $b(t)$ over the discretization in time $t$. This observation implies that $T_{e}=x_{e} / \mu$ also in the case where $b(t)$ is bounded and has a bounded continuous derivative. (Alternatively, instead of applying results of the Euler-Maruyama method, it can be shown that as the piecewise constant approximation of $b(t)$ becomes finer, convergence in mean square takes place which, consequently, implies convergence of the mean first exit time [17].)

Upper and lower bounds for the mean first exit time are derived for the general case of (1.1) in the next theorem.

Theorem 2.3. Assume that $a(t)>0$ for $t>0$ and that $a(t)$ and $b(t)$ are bounded and have bounded continuous first derivatives for $t \in(0, \infty)$. Let $\mu_{1}=\inf _{t>0} a(t)$ and $\mu_{2}=\sup _{t>0} a(t)$. Then, the mean exit time, $T_{e}$, to problem (1.1) satisfies

$$
x_{e} / \mu_{2} \leq T_{e} \leq x_{e} / \mu_{1}
$$

Proof. The proof follows from Proposition 2.1 and Theorem 2.2.

\section{EXAMPLES}

Several examples are presented to illustrate the results of the previous two sections and to show how the results are useful in applications.

\subsection{EXAMPLE A}

This example illustrates use of Proposition 1.1, Theorem 2.2, and Theorem 2.3. Three different exit-time problems, summarized in Table 1, are considered. 


\begin{tabular}{|l|l|l|l|l|}
\hline Problem & $a(t)$ & $b(t)$ & $x_{e}$ & Relation satisfied by $T_{e}$ \\
\hline A1 & $4 \tanh (t)$ & $\sqrt{8 \tanh (t)}$ & 1 & $T_{e}=\int_{0}^{\infty} h(\tau) p\left(\tau ; x_{e}, q\right) d \tau$ \\
A2 & 3 & $\sqrt{6+2 \sin (t)}$ & 3 & $T_{e}=x_{e} / 3$ \\
A3 & $3+\cos (t)$ & $\sqrt{6+2 \sin (t)}$ & 3 & $x_{e} / 4<T_{e}<x_{e} / 2$ \\
\hline
\end{tabular}

Table 1: Problems llustrate Proposition 1.1 and Theorems 2.2 and 2.3

In problem A1, $a(t) / \frac{1}{2} b^{2}(t)=q=1$ so Proposition 1.1 can be applied and it follows that $T_{e}=\int_{0}^{\infty} \cosh ^{-1}(\exp (\tau / 4)) p(\tau ; 1,1) d \tau$. Evaluating this integral gives $T_{e}=0.654$. In comparison, simulations with the Euler-Maruyama method applied to the original problem give $T_{e} \approx 0.66$ which is in agreement. In problem A2, the drift coefficient $a(t)=\mu=3$ is constant and the diffusion coefficient is smooth and bounded. Applying Theorem 2.2 gives $T_{e}=x_{e} / \mu=1$. In problem A3, the drift and diffusion coefficients are smooth and bounded. Applying Theorem 2.3 gives bounds on the mean exit time of $x_{e} / \mu_{2}<T_{e}<x_{e} / \mu_{1}$ and, hence, $0.75<T_{e}<1.5$. Numerical simulations using the Euler-Maruyama method give $T_{e} \approx 0.83$ for problem A3 which agrees with the bounds in Theorem 2.3.

\subsection{EXAMPLE B}

The two-sided exit time problem is briefly considered in the case when $\gamma(t)$ is constant and compared with the one-sided problem. The two-sided exit problem is defined by the Itô stochastic differential equation

$$
d x(t)=a(t) d t+b(t) d W(t)
$$

where $W(t)$ is a standard Wiener process, $x(0)=0$, and exit occurs at the first time $t_{e}$ when either $x\left(t_{e}\right)=x_{1}^{e}$ or $x\left(t_{e}\right)=x_{2}^{e}$ where $x_{1}^{e}<0$ and $x_{2}^{e}>0$ are constants. Suppose that $\gamma(t)=\frac{a(t)}{\frac{1}{2} b^{2}(t)}=q$. Let $\tau=\int_{0}^{t} a(z) d z$ and let $\tau_{e}(y)$ be the mean exit time for a particle initially at $y$. Integrating the backward equation (1.7) over $\tau$ from 0 to $\infty$ gives [2]:

$$
-1=\frac{d \tau_{e}(y)}{d y}+\frac{1}{q} \frac{d^{2} \tau_{e}(y)}{d^{2} y}
$$




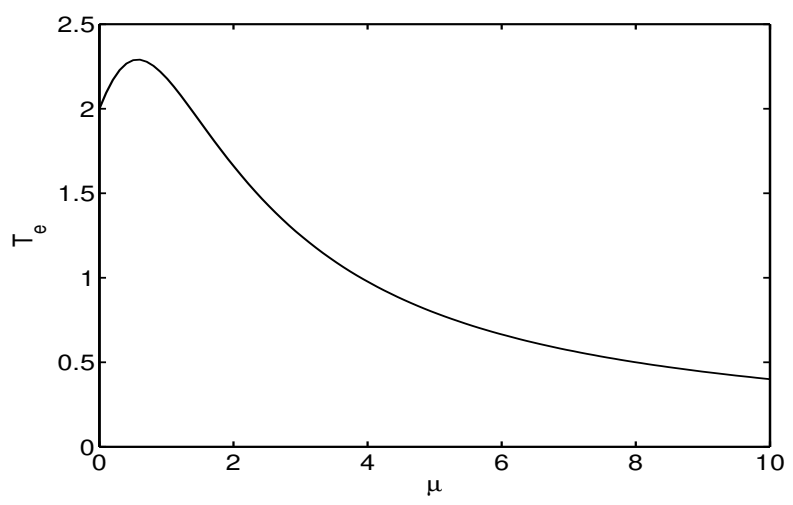

Figure 1: Mean exit time versus drift coefficient for a two-sided problem

with $\tau_{e}\left(x_{1}^{e}\right)=\tau_{e}\left(x_{2}^{e}\right)=0$. This equation yields the result, analogous to that in Proposition 1.1, that

$$
\mathbb{E}\left(\int_{0}^{t_{e}} a(z) d z\right)=\tau_{e}(0)=\frac{x_{1}^{e}\left(1-\exp \left(-q x_{2}^{e}\right)\right)+x_{2}^{e}\left(\exp \left(-q x_{1}^{e}\right)-1\right)}{\exp \left(-q x_{1}^{e}\right)-\exp \left(-q x_{2}^{e}\right)}
$$

However, similar results such as Proposition 2.1 and Theorems 2.2 and 2.3 do not hold for the two-sided problem. To see this, let $\frac{1}{2} b^{2}(t)=1$ and $a(t)=\mu$ so $q=\mu$ in (3.2) and, for convenience, let $x_{1}^{e}=-1$ and $x_{2}^{e}=4$. Then, $T_{e}$ satisfies the equation

$$
T_{e}=\frac{-5+\exp (-4 \mu)+4 \exp (\mu)}{\mu(\exp (\mu)-\exp (-4 \mu))} .
$$

A graph of this equation is given in Figure 1 which shows the complicated nature of the mean exit time as the drift coefficient $\mu$ varies even though the diffusion coefficient is held fixed. The exit-time behavior for the two-sided problem differs from that of the one-sided problem.

In addition, as exits from the two-sided problem occur before or at the same time as those from the one-sided problem, an upper bound on the mean exit time for a two-sided problem can be obtained by considering the mean exit time for the corresponding one-sided problem. 


\subsection{EXAMPLE C}

A persistence time problem, described in [4], is examined where the population size $y(t)$ satisfies

$$
d y(t)=r(t) y(t) d t
$$

with $y(0)=y_{0}$ and where $r(t)$ is the per capita population growth rate. In a randomly varying environment, $r(t)$ varies with time $t$ and experiences random changes. The persistence time, defined as the time until the population decreases below unity, is studied. Two forms for the variability in $r(t)$ are hypothesized. First, a linear function of white noise is assumed where $r(t)$ has the form

$$
r(t)=\alpha_{1}+\beta_{1} \frac{d W(t)}{d t},
$$

with $W(t)$ a standard Wiener process, $\alpha_{1}<0$, and $\beta_{1}>0$. For this case, the population size $y(t)$ then satisfies the Itô stochastic differential equation

$$
d y(t)=\alpha_{1} y(t) d t+\beta_{1} y(t) d W(t) .
$$

In the second hypothesis, an Ornstein-Uhlenbeck mean-reverting process [4] is assumed for $r(t)$ and so $r(t)$ and $y(t)$ satisfy the system

$$
\left\{\begin{array}{l}
d r(t)=\gamma\left(\alpha_{2}-r(t)\right) d t+\beta_{2} d W(t), \\
d y(t)=r(t) y(t) d t
\end{array}\right.
$$

with $r(0)=\alpha_{2}, W(t)$ a standard Wiener process, $\gamma>0, \alpha_{2}<0$, and $\beta_{2}>0$. To understand the difference in the two models with regard to persistence time, let $\bar{r}=\frac{1}{T} \int_{0}^{T} r(s) d s$ be the time-averaged growth rate for large time $T$, then

$$
\mathbb{E}(\bar{r})=\alpha_{1} \text { for model (3.6) and } \mathbb{E}(\bar{r})=\alpha_{2} \text { for model (3.7), }
$$

$$
\text { where } \alpha_{1}, \alpha_{2}<0 \text {. }
$$

The mean persistence time is now examined for these two models. First, Itô's formula $[10,13,14]$ is used to convert model (3.6) into a simpler exit time problem. Let $z_{1}(t)=\log (y(t))$. Then, using Itô's formula, $z_{1}(t)$ satisfies the exit time problem

$$
d z_{1}(t)=\left(\alpha_{1}-\beta_{1}^{2} / 2\right) d t+\beta_{1} d W(t)
$$


with $z_{1}(0)=\log \left(y_{0}\right)$ and exit occurring at $t_{e}$ when $z_{1}\left(t_{e}\right)=0$. By Proposition (1.1) and considering the translated variable $\log \left(y_{0}\right)-z_{1}(t)$, the mean exit time satisfies

$$
T_{e}=\log \left(y_{0}\right) /\left(-\alpha_{1}+\beta_{1}^{2} / 2\right) .
$$

The second problem (3.7) is simplified by letting $z_{2}(t)=\int_{0}^{t} r(s) d s$. As

$$
r(t)=\alpha_{2}+\exp (-\gamma t) \int_{0}^{t} \beta_{2} \exp (\gamma u) d W(u)
$$

it can be shown that $z_{2}(t)$ satisfies

$$
z_{2}(t) \sim \mathbb{N}\left(\alpha_{2} t, \frac{\beta_{2}^{2}}{\gamma^{2}}\left(t-\frac{2}{\gamma}(1-\exp (-\gamma t))+\frac{1}{2 \gamma}(1-\exp (-2 \gamma t))\right)\right)
$$

implying that $z_{2}(t)$ solves the Itô SDE

$$
d z_{2}(t)=\alpha_{2} d t+b(t) d W(t) \quad \text { with } \quad z(0)=0
$$

where $b^{2}(t)=\frac{\beta_{2}^{2}}{\gamma^{2}}(1-\exp (-\gamma t))^{2}$. As exit occurs when population size $y(t)$ reaches unity, or $y_{0} \exp \left(\int_{0}^{t} r(s) d s\right)=1$, then exit occurs at the identical time when $z_{2}(t)=-\log \left(y_{0}\right)$. Thus, an equivalent problem for the persistence time for the $\operatorname{SDE}$ system (3.7) when $y(0)=y_{0}$ and exit occurs at $y(t)=1$ is to study the persistence time for the scalar $\operatorname{SDE}(3.10)$ when $z_{2}(0)=0$ and exit occurs at $z_{2}(t)=-\log \left(y_{0}\right)$. Persistence-time problem (3.10) is a diffusion process with one absorbing boundary and purely time-dependent drift and diffusion coefficients. By Theorem (2.2), the mean persistence time is exactly equal to $-\log \left(y_{0}\right) / \alpha_{2}$. In particular, as $\alpha_{2} \rightarrow 0$ and a pure diffusion process is approached, the mean persistence time for (3.10) goes to infinity. Correspondingly, the mean persistence time for (3.7) goes to infinity as $\alpha_{2} \rightarrow 0$.

It is observed that the two growth rate models (3.5) and (3.7) give different interpretations with respect to how the parameters influence the mean persistence time. Recall that $\mathbb{E}(\bar{r})$ is equal to the average growth rate over a large time interval $T$ and $\mathbb{E}(\bar{r})=\alpha_{1}$ for (3.6) and $\mathbb{E}(\bar{r})=\alpha_{2}$ for (3.7). For (3.6), the mean persistence time goes to the constant value $2 \log \left(y_{0}\right) / \beta_{1}^{2}$ as $\mathbb{E}(\bar{r}) \rightarrow 0$, whereas for (3.7), the mean persistence time goes to $\infty$ as $\mathbb{E}(\bar{r}) \rightarrow 0$. The interpretation of mean persistence is different for the two different hypotheses. 


\section{SUMMARY}

In the present investigation, exact mean exit times are derived for the onedimensional first-passage time problem with one-sided exit for a constant drift coefficient but a time-dependent diffusion coefficient. When the drift and diffusion coefficients both vary with time, bounds on mean first exit time are derived. Several examples are described that illustrate the usefulness of the results.

\section{REFERENCES}

[1] E. J. Allen, L. J. S. Allen, H. Schurz, A comparison of persistence-time estimation for discrete and continuous stochastic population models that include demographic and environmental variability, Mathematical Biosciences 196 (2005), 14-38.

[2] E. J. Allen, Modeling With Itô Stochastic Differential Equations, Springer, Dordrecht, 2007.

[3] E. J. Allen, A stochastic analysis of power doubling time for a subcritical system, Stochastic Analysis and Applications 31 (2013), 528-537.

[4] E. Allen, Environmental variability and mean-reverting processes, Discrete and Continuous Dynamical Systems Series B 21 (2016), 2073-2089.

[5] L. J. S. Allen, E. J. Allen, A comparison of three different stochastic population models with regard to persistence time, Theoretical Population Biology 68 (2003), 439-449.

[6] L. J. S. Allen, An Introduction to Stochastic Processes with Applications to Biology, Second Edition, CRC Press, Chapman \& Hall Publishers, Boca Raton, 2010.

[7] B. Bouchard, S. Geiss, E. Gobet, First time to exit of a continuous Itô process: general moment estimates and $L 1$-convergence rate for discrete time approximations, Bernoulli, Bernoulli Society for Mathematical Statistics and Probability, 2015, hal-00844887v2. 
[8] R. Chicheportiche, J.-P. Bouchaud, Some applications of first-passage ideas to finance, in: First-Passage Phenomena and Their Applications, (Ed: R. Metzler, G. Oshanin, S. Redner), World Scientific Publishing Company, Singapore, 2014.

[9] J. L. Folks and R. S. Chhikara, The inverse Gaussian distribution and its statistical application - a review, Journal of the Royal Statistical Society: Series B 40 (1978), 263-289.

[10] T. C. Gard, Introduction to Stochastic Differential Equations, Marcel Decker, New York, 1987.

[11] E. Gobet and S. Menozzi, Stopped diffusion processes: Boundary corrections and overshoot, Stochastic Processes and their Application 120 (2010), 130-162.

[12] D. J. Higham, X. Mao, M. Roj, Q. Song, and G. Yin, Mean exit times and the multilevel Monte Carlo method, SIAM/ASA Journal on Uncertainty Quantification 1 (2013), 2-18.

[13] P. E. Kloeden, E. Platen, H. Schurz, Numerical Solution of SDE Through Computer Experiments, Springer, Berlin, 1994.

[14] A. G. Ladde, G. S. Ladde, An Introduction to Differential Equations: Stochastic Modeling, Methods and Analysis, Volume 2, World Scientific Publishing Company, Singapore, 2013.

[15] A. Molini, P. Talkner, G. G. Katul, A. Porporato, First passage time statistics of Brownian motion with purely time dependent drift and diffusion, Physica A: Statistical Mechanics and its Applications 390 (2011), 1841-1852.

[16] J. B. Roberts, First-passage probabilities for randomly excited systems: diffusion methods, Probabilistic Engineering Mechanics 1 (1986), 66-81.

[17] M. Seesselberg and F. Petruccione, An improved algorithm for the estimation of the mean first passage time of ordinary stochastic differential equations, Computer Physics Communications 74 (1993), 247-245. 
[18] W. D. Sharp and E. J. Allen, Numerical solution of first passage time problems using an approximate Chapman-Kolmogorov relation, Probabilistic Engineering Mechanics 13 (1998), 233-241.

[19] C. Skiadas and C. H. Skiadas, Development, simulation, and application of first-exit-time densities to life table data, Communications in Statistics - Theory and Methods 39 (2010), 444-451. 